\title{
Langerhanszell-Histiozytose unter dem Bild einer Folliculitis decalvans und Intertrigo*
}

\section{Langerhans Cell Histiocytosis Mimicking Folliculitis Decalvans and Intertrigo}

Autoren

S. Nasifoglu, J. Welzel

Institut

Klinik für Dermatologie und Allergologie, Klinikum

Augsburg

Korrespondenzadresse

Dr. med. Suzan Nasifoglu, Klinik für Dermatologie und

Allergologie, Klinikum Augsburg, Sauerbruchstraße 6,

86179 Augsburg

Suzan.nasifoglu@gmail.com

\section{ZUSAMMENFASSUNG}

Die Langerhanszell-Histiozytose ist eine seltene Erkrankung unklarer Ätiologie, die durch eine Proliferation histiozytärer Zellen in verschiedenen Geweben gekennzeichnet ist. Vor wenigen Jahren wurde entdeckt, dass ein Großteil der LCHZellen eine BRAF-Mutation aufweist. Eine neoplastische Genese ist demnach denkbar. Da mehrere Organe durch die proliferierende Zellen betroffen sein können, sind die Beschwerden der Langerhanszell-Histiozytose vielgestaltig. Das am häufigsten betroffene Organ bei der erwachsenen Langerhanszell-Histiozytose ist die Lunge, gefolgt von den Knochen. In 1/3 der Fälle ist die Haut betroffen, sodass dem Dermatologen bei der Diagnosefindung eine ausgesprochen wichtige Rolle zukommt. Wegweisende Befunde sind die Trias erosive, ekzemartige Hautveränderungen am Kapillitium sowie in den Intertrigines in Kombination mit einem Diabetes insipidus. Die Diagnose wird letztlich histologisch gestellt. Typisch sind dichte Infiltrate, bestehend aus großen Zellen mit bohnenförmigem Zellkern, die immunhistochemisch durch Nachweis von CD1a-Antigen auf der Zelloberfläche charakterisiert sind.

Therapeutisch werden neben topischen Steroiden, PUVA, systemischen Immunsuppressiva auch teilweise Polyche-

\footnotetext{
* Fallvorstellung am 1.05.2016, 24. Jahrestagung der Arbeitsgemeinschaft Dermatologische Histologie, Hildesheim, und am 6. 07. 2016, Frühjahrsitzung der Münchner Dermatologischen Gesellschaft e.V. München.
}

motherapien sowie chirurgische und auch strahlentherapeutische Maßnahmen angewandt. Aufgrund der neuesten Erkenntnisse der bestehenden BRAF-Mutation wird derzeit auch der Einsatz von BRAF-Inhibitoren diskutiert.

Bei lokalisierten Formen der LCH ist die Prognose günstig. Der Ausgang der disseminierten Form ist abhängig vom Ausmaß des Organbefalls sowie vom Alter der Patienten. In seltenen Fällen wurden Entwicklungen zu akuten myeloischen Leukämien beobachtet. Aufgrund der Seltenheit der Erkrankungen hat sich bisher kein systematischer und interdisziplinärer Ansatz zur Diagnosestellung und Therapie ebenso wie kein langfristiges Nachsorgekonzept etabliert.

\section{ABSTRACT}

Langerhans cell histiocytosis (LCH) is a rare disease of unknown etiology characterized by the infiltration of one or more organs by large mononuclear cells. A few years ago, BRAF mutation was discovered in LCH cells. A neoplastic genesis is therefore conceivable. Although the features of this disease are well described in children, they remain poorly defined in adults.

The most common organ involved is the lung followed by bone and skin. As nearly every organ can be affected, health problems can be very unspecified. Typical symptoms are erosive, eczematous skin lesions of the scalp and intertrigines in combination with a diabetes insipidus. Diagnosis of LCH is usually made by skin biopsy performed because of persistent skin lesions. Dense infiltrates consisting of a number of cells with a bean-shaped nucleus, which immunohistochemically provide detection of CD1a antigen on the cell surface are typically.

In addition to topical steroids, PUVA and systemic immunosuppressants, partial polychemotherapy and surgical as well as radiotherapeutic measures are used therapeutically. On the basis of the latest findings from the BRAF mutation, the use of BRAF inhibitors is currently being discussed.

In localized forms of LCH, prognosis is good. The outcome of the disseminated form depends on the extent of the organ involvement and the age of the patients. In some rare cases, developments into acute myelogenous leukemia have been observed.

Due to the rarity of the diseases, no systematic and interdisciplinary approach to diagnosis and therapy as well as no long-term follow-up concept has been established. 


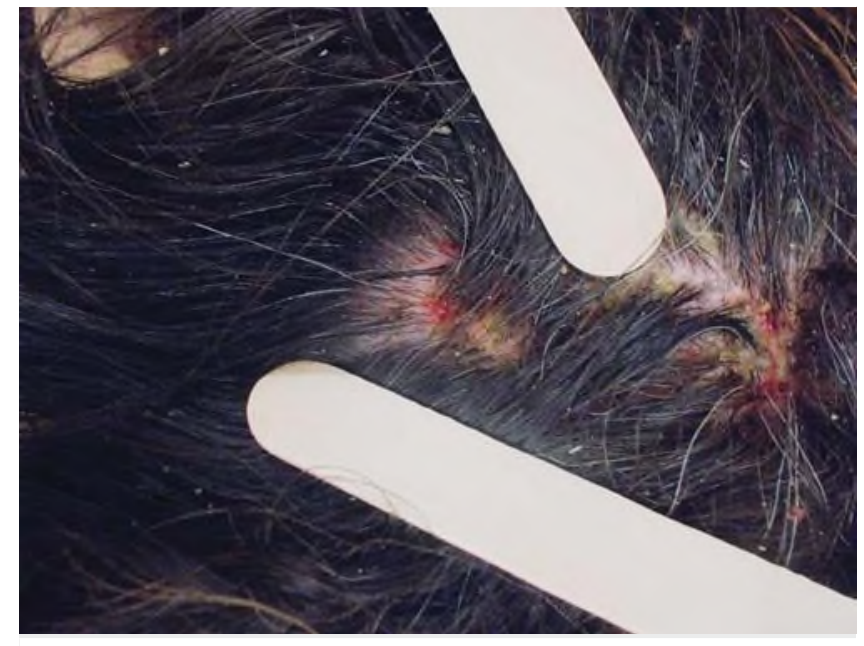

- Abb. 1 Großflächig konfluierende Erytheme mit schmerzhaften, teils krustös belegten, gruppiert stehenden Papeln am Kapillitium bei Langerhanszell-Histiozytose.

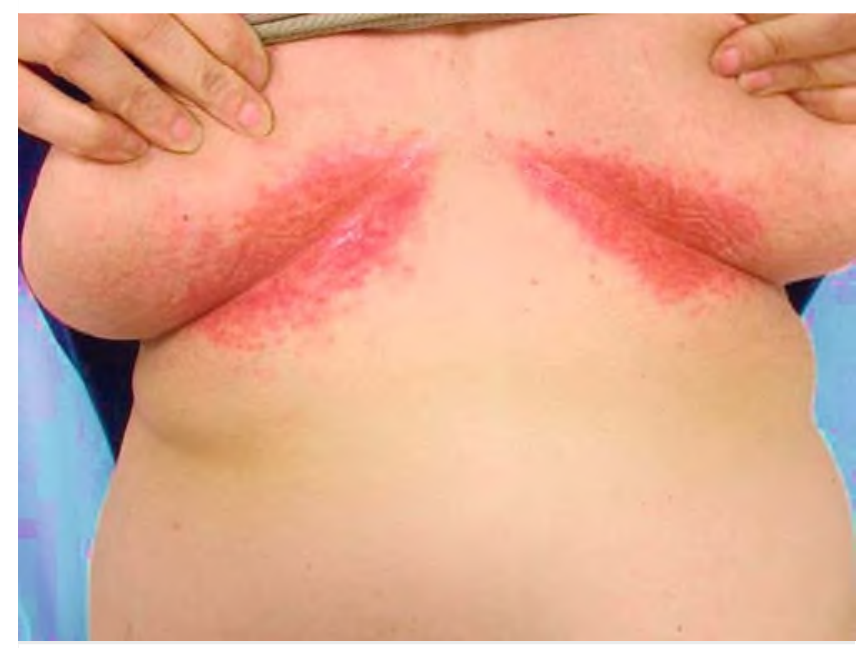

- Abb. 2 Scharf begrenzte Erytheme submammär beidseits bei Langerhanszell-Histiozytose.

\section{Kasuistik}

\section{Anamnese}

Eine 47-jährige Patientin stellte sich mit seit etwa einem Jahr bestehender schuppender Kopfhaut einhergehend mit Juckreiz, Schmerzen und Brennen vor. Bei zuvor schon geäußertem Verdacht auf eine Follikulitis decalvans erfolgte eine antibiotische Therapie sowie die topische Anwendung von Steroiden. Beide Therapieansätze waren sine effectu.

Seit einem Monat waren zusätzlich ausgeprägte Hautveränderungen im Bereich beider Leisten, der Rima ani sowie submammär beidseits aufgetreten. Rezente Infekte, B-Symptomatik sowie Krankheitsgefühl bestanden nicht. Die Patientin berichtete, immer wieder unter Mittelohrentzündungen zu leiden.

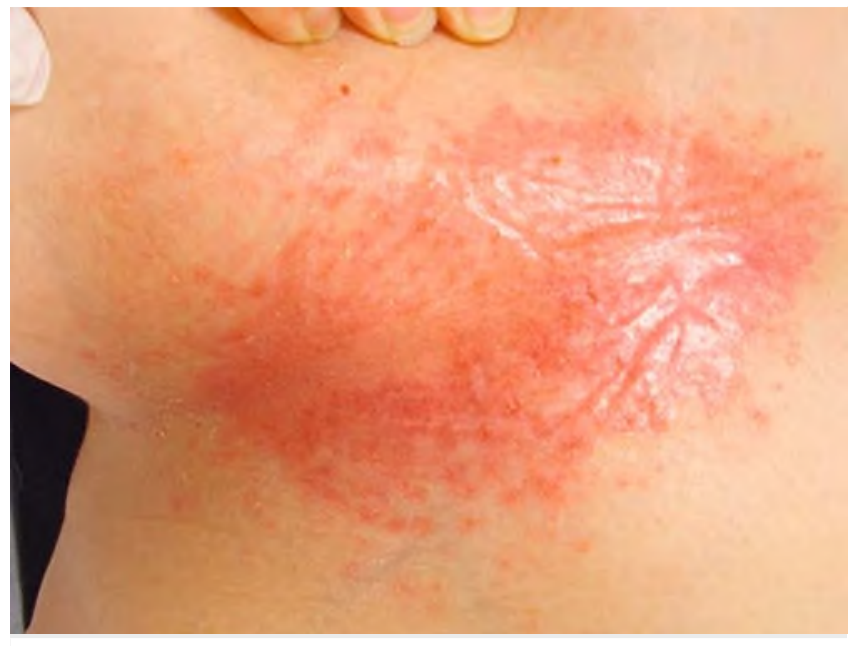

- Abb. 3 Scharf begrenztes, glänzendes Erythem mit peripher follikulär gebundenen Papeln submammär rechts bei Langerhanszell-Histiozytose.

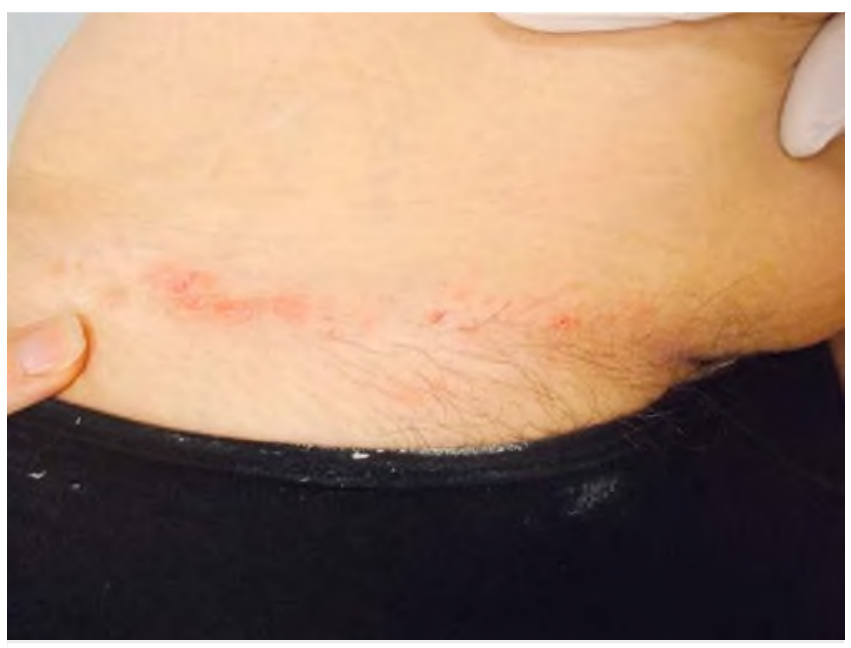

Abb. 4 Vereinzelte Erosionen auf scharf begrenzten Erythemen inguinal links bei Langerhanszell-Histiozytose.

\section{Befunde}

Am Kapillitium zeigten sich sowohl disseminiert als auch gruppiert stehende Papeln, teils krustös belegt, sowie großflächig konfluierende Erytheme ( $\boldsymbol{A} \mathbf{A b} \mathbf{b} . \mathbf{1})$. Submammär beidseits waren zudem scharf begrenzte Erytheme mit randbetonten Pusteln sowie inguinal als auch an der Rima ani scharf begrenzte Erytheme mit disseminierten, maximal $2 \times 3 \mathrm{~mm}$ großen Erosionen mit rötlichem Randsaum zu sehen ( $\triangleright$ Abb. $2-4$ ). Zudem sei bei der Patientin ein zentraler Diabetes insipidus bekannt. Dieser sei vor über 10 Jahren nach langer Beschwerdeepisode durch einen Endokrinologen diagnostiziert worden. Unter der derzeitigen Therapie mittels eines ADH-Derivates sei die Patientin diesbezüglich weiterhin beschwerdefrei. Des Weiteren bestand eine mikrozytäre hypochrome Anämie sowie eine rezidivierende Otitis media. Ansonsten waren keine wesentlichen Vorerkran- 


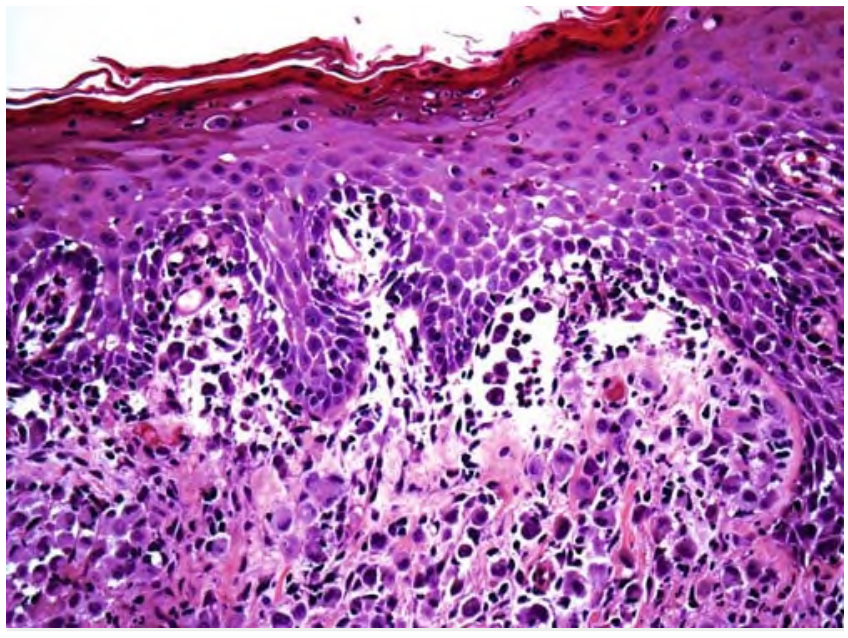

- Abb. 5 Histologie Kapillitium bei Langerhanszell-Histiozytose, H\&E-Färbung, Vergrößerung 20×. Deutlicher Epidermo- und Follikulotropismus. Infiltrat bestehend aus großen Zellen mit bohnenförmigem Zellkern.

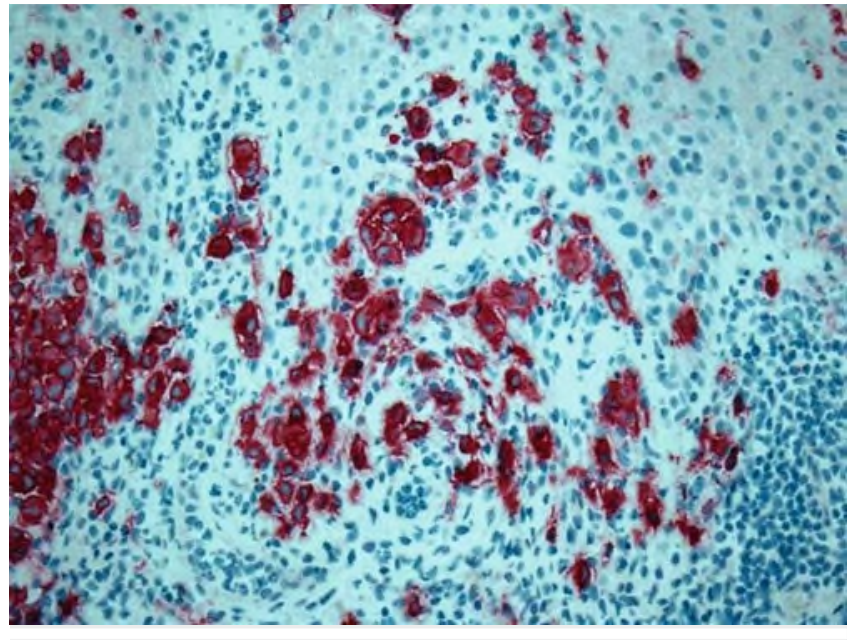

Abb. 6 Immunhistochemischer Nachweis von CD1a-Antigen auf der Zelloberfläche der infiltrierten Bereiche, Vergrößerung 20×, Langerhanszell-Histiozytose.

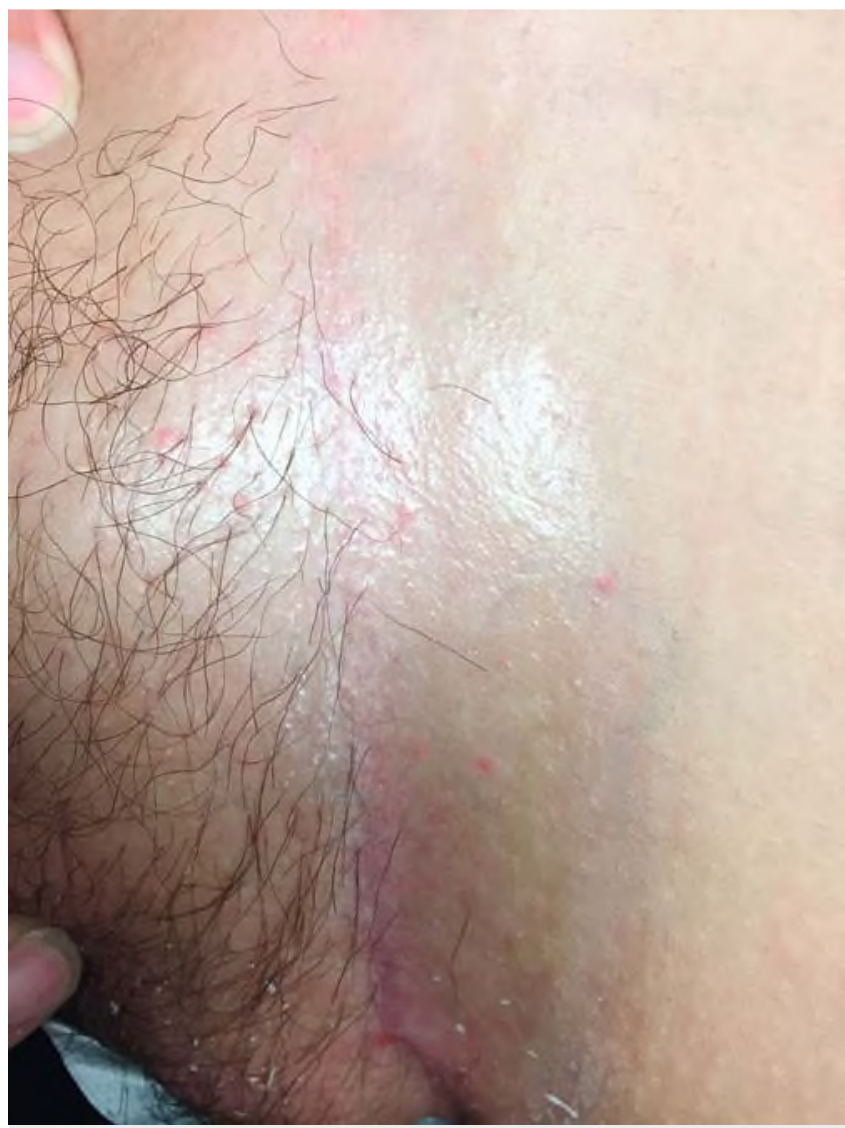

Abb.7 Langerhanszell-Histiozytose, Hautbefund inguinal links 9 Monate nach Beginn der Therapie mit Methotrexat.
Die Langerhanszell-Histiozytose (LCH), früher auch Histiozytosis $X$ genannt, ist durch eine Proliferation histiozytärer Zellen in verschiedenen Geweben gekennzeichnet. Im Kindesalter beträgt die Inzidenz 3-5/1000 000 jährlich. Bei Erwachsenen tritt das Krankheitsbild deutlich seltener auf [1]. kungen bekannt. Die Patientin befand sich in einem guten Gesundheitszustand.

Die histologische Aufarbeitung zeigte eine kompakte Hyperkeratose, eine fokal erodierte atrophe Epidermis, ein die Dermis durchsetzendes, dichtes Infiltrat sowie einen immunhistoche-

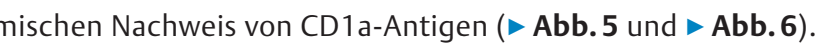

Der BRAF-Mutationsstatus war negativ. In dem durchgeführten PET-CT zeigten sich Mehranreicherungen in den klinisch auffälligen Hautbereichen am Kapillitium, im Genitalbereich, an der Rima ani, an der rechten Leiste sowie auch in den Gehörgängen, aber keine interne Manifestation.

Eine ebenfalls durchgeführte Röntgen-Thorax-Untersuchung, eine Abdomensonografie, ein kraniales MRT sowie eine Lungenfunktionsuntersuchung zeigten keine relevanten Auffälligkeiten.

\section{Therapie und Verlauf}

Basierend auf den Ergebnissen von Steen et al. erfolgte bei unserer Patientin die Initiierung einer systemischen immunsuppressiven Therapie mit Methotrexat nach Ausschluss möglicher Kontraindikationen [12]. Die Kontrolluntersuchungen erfolgten alle 3 Monate, zuletzt 9 Monate nach Beginn der Therapie. Es konnte bisher eine signifikante Regredienz des Hautbefundes sowie eine deutliche Linderung der Beschwerdesymptomatik der Patientin gezeigt werden ( $\triangleright$ Abb.7-9).

\section{Diskussion}




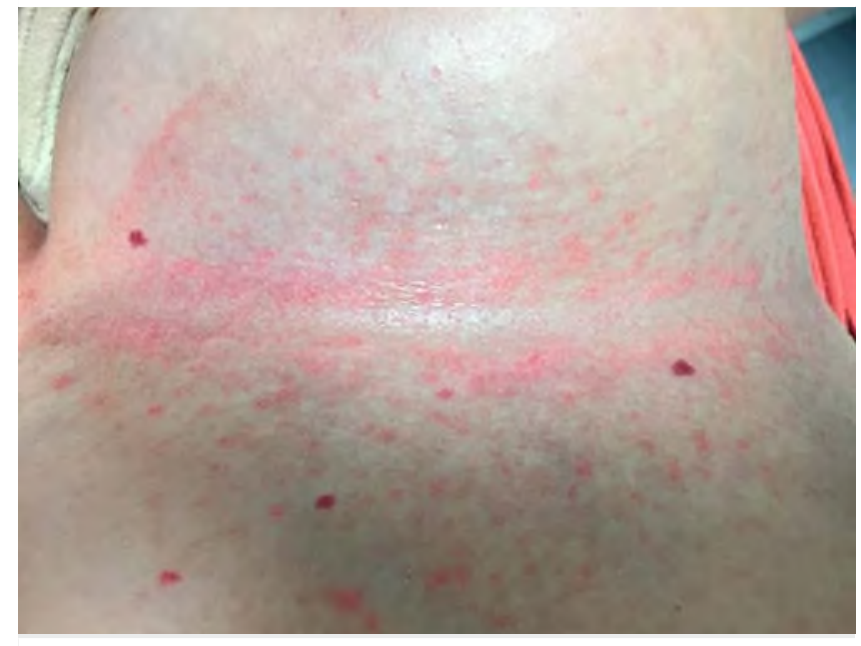

- Abb. 8 Langerhanszell-Histiozytose, Hautbefund submammär links 9 Monate nach Beginn der Therapie mit Methotrexat.

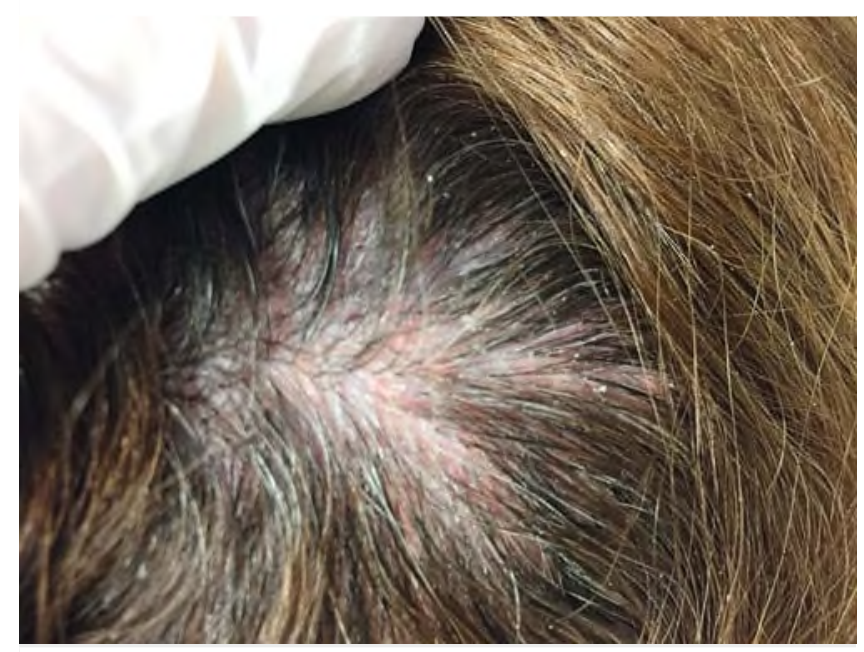

- Abb.9 Langerhanszell-Histiozytose, Hautbefund Kapillitium 9 Monate nach Beginn der Therapie mit Methotrexat.

Die proliferierenden dendritischen Zellen unterscheiden sich ultrastrukturell nicht von Langerhanszellen der Epidermis und exprimieren demnach ebenfalls den Haupthistokompatibilitätskomplex CD1a. Als Pathomechanismus wird eine Zytokinimbalance zwischen T-Zellen und Langerhanszellen beschrieben, die zu einem interzellulären Kommunikationsdefekt führt. Die Ätiologie ist bisher nicht bekannt [10]. Vor wenigen Jahren wurde entdeckt, dass ein Großteil der LCH-Zellen eine BRAFMutation aufweist. Eine neoplastische Genese ist demnach wahrscheinlich und ist derzeit Gegenstand aktueller Studien [9].

Das klinische Spektrum der LCH ist äußerst vielgestaltig und reicht von einem unilokulären (Knochen, Haut, Lymphknoten, Lunge) oder multilokulären Befall eines Organs oder Organsystems bis hin zum disseminierten Befall mehrerer Organe sowie von benignen bis hin zu malignen Verläufen [8]. Es wurde bereits eine Entwicklung von akuten myeloischen Leukämien sowie in Einzelfällen auch von Lymphomen beobachtet [5, 7,
11]. Das am häufigsten betroffene Organ ist die Lunge, gefolgt von den Knochen und anschließend der Haut [2], wobei die höchste Mortalität bei isoliertem Lungenbefall beobachtet wird [3]. Da die Haut in mindestens $1 / 3$ der Fälle betroffen ist, kommt dem Dermatologen bei der Diagnosestellung eine ausgesprochen wichtige Rolle zu.

Diagnostisch wegweisend sind erosive, ekzemartige Hautveränderungen am Kapillitium sowie in den Intertrigines in Kombination mit einem Diabetes insipidus [6]. Der Diabetes insipidus ist die häufigste endokrinologische Manifestation und betrifft $30 \%$ der Patienten, wie es auch bei unserer Patientin der Fall war. Als Ursache findet sich eine Läsion im Bereich des Hypophysenhinterlappens [1]. Die Manifestation des Diabetes insipidus kann, wie es bei unserer Patientin der Fall war, vor oder nach der Diagnose der LCH erfolgen.

Da bisher keine kontrollierten Therapiestudien vorliegen, sind die Therapiemöglichkeiten bei Erwachsenen sehr begrenzt. Neben topischen Steroiden, PUVA und systemischen Immunsuppressiva werden teilweise auch Polychemotherapien sowie chirurgische und auch strahlentherapeutische Ansätze angewandt. BRAF-V600E-Mutationen in LCH wurden bereits beschrieben, sodass der Einsatz von BRAF-Inhibitoren derzeit diskutiert wird [4].

Bei lokalisierten Formen der LCH kann die Prognose insgesamt als günstig bezeichnet werden. Die Prognose der disseminierten Form ist abhängig vom Ausmaß des Organbefalls sowie vom Alter der Patienten. Aufgrund der Seltenheit der LCH fehlt bislang ein systematischer und interdisziplinärer Ansatz zur Diagnosestellung und Therapie ebenso wie ein langfristiges Nachsorgekonzept. Letztlich sind interdisziplinäre Leitlinien zur Standardisierung von Diagnose und Therapie und der weitere Aufbau eines zentralen Registers bei der LCH des Erwachsenen dringend notwendig [8].

Interessenkonflikt

Die Autorinnen geben an, dass kein Interessenkonflikt besteht. Dieser Beitrag beinhaltet keine Studien an Menschen oder Tieren.

Literatur

[1] Choi J-E, Lee HR, Ohn JH et al. Adult Multisystem Langerhans Cell Histiocytosis Presenting with Central Diabetes Insipidus Successfully treated with Chemotherapy. Endocrinol Metab 2014; 29: 394 - 399

[2] https://www.cancer.gov/types/langerhans/hp/langerhans-treatment-pdq

[3] Arico M, Girschikofsky M, Genereau T et al. Langerhans cell histiocytosis in adults. Report from the International Registry of the Histiocyte Society. Eur J Cancer 2003; 39: 2341 - 2348

[4] Berres ML, Lim KP, Peters T et al. BRAF-V600E expression in precursor versus differentiated dendritic cells defines clinically distinct $\mathrm{LCH}$ risk groups. The Journal of experimental medicine 2014; 211: 669-683

[5] Blazewicz I, Sokolowska-Wojdylo M, Piekarska A et al. Langerhans cell histiocytosis followed by folliculotropic mycosis fungoides. Postepy Dermatol Alergol 2017; 34: 273-275

[6] Blind A, Sachs C, Blaison G et al. Scalp papules revealing Langerhans cell histiocytosis. Ann Dermatol Venereol 2017; 144: 732 - 735 
[7] Egeler RM, Neglia JP, Arico M et al. Acute leukemia in association with Langerhans cell histiocytosis. Med Pediatr Oncol 1994; 23: 81-85

[8] Fichter J, Doberauer C, Seegenschmiedt H. Langerhans-Zellhistiozytose des Erwachsenen - Eine interdisziplinäre Herausforderung. Dtsch Arztebl 2007; 104: A-2347 - A-2353

[9] Milne P, Bigley V, Bacon CM et al. Hematopoietic origin of Langerhans cell histiocytosis and Erdheim-Chester disease in adults. Blood 2017; 130: $167-175$
[10] Ruzicka T, Evers ]. Langerhanszell-Histiozytose: Klinische Verlaufsformen und Therapie. Hautarzt 2003; 54: 148 - 155

[11] Safaei A, Bagheri M, Shahryari J et al. Langerhans cell histiocytosis followed by hodgkin lymphoma: a case report. Iran J Med Sci 2015; 40: $282-286$

[12] Steen AE, Steen KH, Bauer R et al. Successful treatment of cutaneous Langerhans cell histiocytosis with low-dose methotrexate. $\mathrm{Br}$ J Dermatol 2001; 145: $137-140$ 